\title{
In situ Characterization of Laser Powder Bed Fusion Using High-Speed Synchrotron X-ray Imaging Technique
}

\author{
Niranjan Parab ${ }^{1}$, Cang Zhao ${ }^{1}$, Ross Cunningham², Luis I. Escano ${ }^{3}$, Kamel Fezzaa ${ }^{1}$, Anthony Rollett ${ }^{2}$, \\ Lianyi Chen ${ }^{3}$ and Tao Sun ${ }^{*}$ \\ 1. X-ray Science Division, Argonne National Laboratory, Lemont, IL, USA \\ 2. Department of Materials Science and Engineering, Carnegie Mellon University, Pittsburg, PA, USA \\ 3. Department of Mechanical and Aerospace Engineering, Missouri University of Science and \\ Technology, Rolla, MO, USA. \\ * Corresponding author: taosun@anl.gov
}

Additive manufacturing (AM, a.k.a. 3D printing) refers to a suite of transformative technologies that build three-dimensional objects by adding materials layer by layer based on digital design. Compared with conventional manufacturing techniques, AM exhibits many unique advantages, including short supply chain, on-site and on-demand manufacturing of spare units and tools, short design-to-market, less consumption of energy, and less generation of material waste. More importantly, AM almost eliminates tooling constraints, giving us the much-needed freedom to design and build parts with complex geometries and improved performance. Nowadays, there are tens of different AM technologies, which we can categorize into seven families: powder bed fusion, direct energy deposition, binder jetting, material jetting, sheet lamination, vat polymerization, material extrusion, and hybrid. Among all, laser powder bed fusion (LPBF) is the most extensively used technique for 3D printing metals, and has found many applications in the fields of biomedical, aerospace, automobile, and defense.

Owing to substantial investments in the technology from both public and private groups worldwide, LPBF has developed rapidly in the last three decades. Numerous 3D printer manufacturers have emerged, and the technique maturation was seemingly reached. LPBF is a conceptually simple process, but the thermal conditions involved are rather extreme. The local temperature is generally higher than the boiling point of the metal being processed. Meanwhile, the heating and cooling rates are in the order of $10^{6} \mathrm{~K} / \mathrm{s}$, and the thermal gradient can easily reach $10^{3} \mathrm{~K} / \mathrm{mm}$. Given such extreme thermal condition, many highly dynamic and transient physical processes are involved in LPBF. Their complex interplay often leads to structure defects in the build, including but not limited to rough surface, porosity, cracks, residual stress, and undesired phase and grain structures. Therefore, no matter how good the machine design and engineering control are, the success rate of printing a complex part in the first round of attempt remains low. Operator's experience may help identify the initial processing window, but the trial-and-error type effort to further optimize the processing condition is often inevitable. Computer simulation can also help narrow the range of laser parameters for a given material and part geometry. However, most of current LPBF process models are based on indirect observations or post-mortem characterization results, and even the most sophisticated 3D multi-physics models are developed with major assumptions.

At the Advanced Photon Source of Argonne National Laboratory, we recently developed and applied high-speed x-ray imaging technique for in situ studying LPBF processes. (Fig.1) The LPBF simulator consists of a $1070 \mathrm{~nm}$ ytterbium fiber laser source, a galvanometer laser scanner, a vacuum chamber, and two sets of stepping motors. [1-3] The high-flux high-energy photons afforded by the $3^{\text {rd }}$-generation hard $\mathrm{x}$-ray synchrotron facility make it possible to look through dense metallic materials and watch the dynamic structural evolution during the laser-metal interaction. In our x-ray experiments, we demonstrated that 
many dynamic structure parameters in LPBF can be measured in situ with micrometer spatial resolution and microsecond temporal resolution, such as melt pool morphology, melt flow velocity, keyhole dynamics, powder ejection velocity, and solidification rate (Fig. 2). These quantitative information are being used to validate and calibrate different types of numerical models and are encouraging theorists to re-evaluate their hypotheses and assumptions. [4] Also, the real-time observations allow us to understand the mechanisms that govern the formation of different types of defects in LPBF processes.

\section{References:}

[1] C Zhao et al., Scientific Reports 7 (2017), p. 3602.

[2] N Parab et al., Journal of Synchrotron Radiation 25 (2018), p. 1467.

[3] Q Guo et al., Acta Materialia 151 (2018), p. 169.

[4] R Cunningham et al., Science 363 (2019), p. 849.

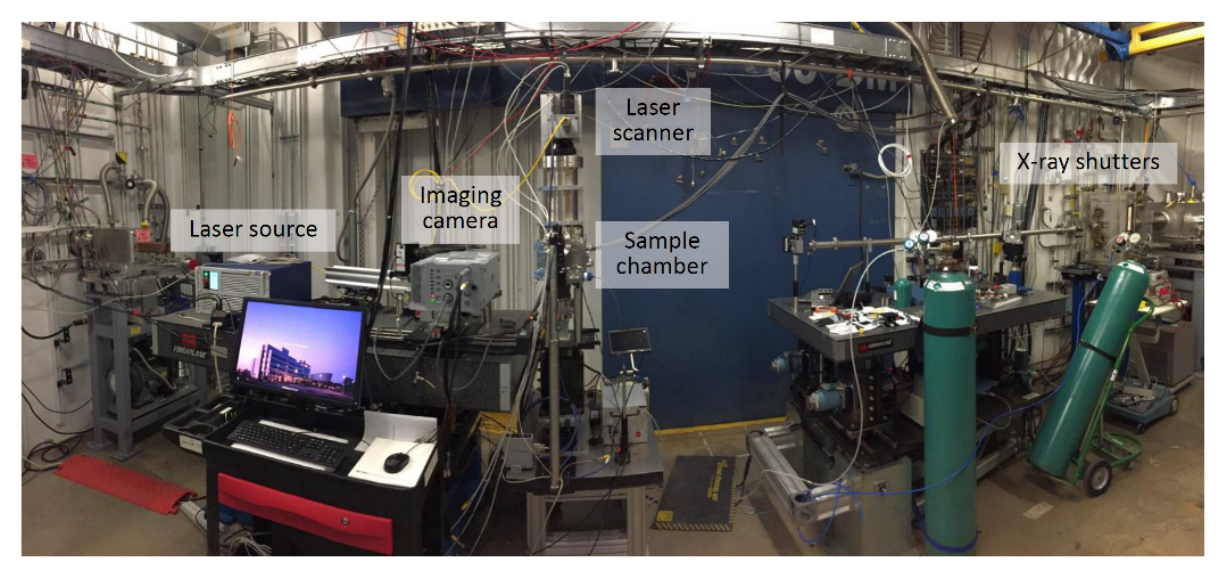

Figure 1. Photograph of the laser additive manufacturing setup at 32-ID-B beamline of the Advanced Photon Source.
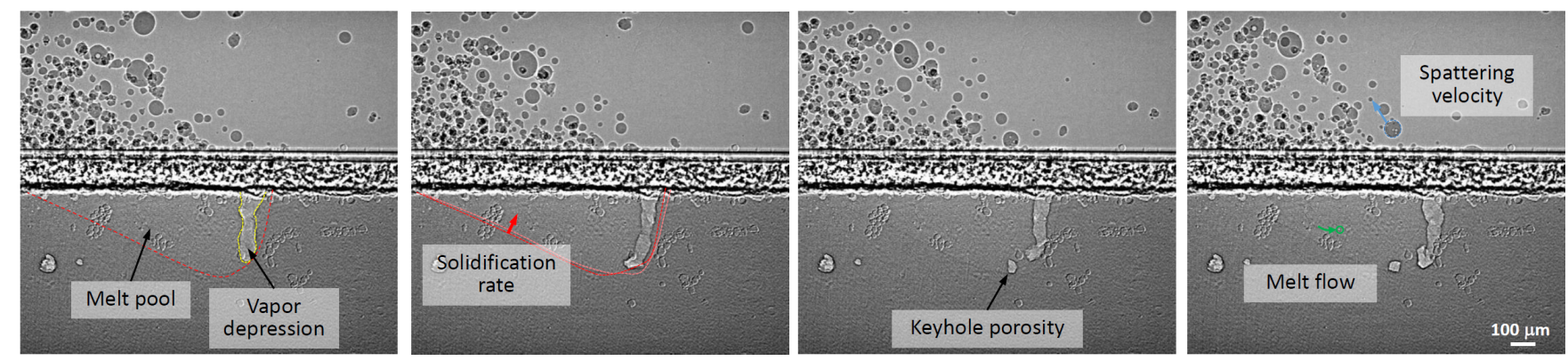

Figure 2. High-speed $\mathrm{x}$-ray image series of laser powder bed fusion process of Al-10Si-Mg. The laser power was set at $520 \mathrm{~W}$ and the scanning speed was $0.6 \mathrm{~m} / \mathrm{s}$. X-ray images were recorded at $30,173 \mathrm{~Hz}$, with an effective exposure time of 100 ps. In the images, quantitative measurements of melt pool and vapor depression morphology, solidification rate, melt flow velocity, and particle spattering velocity are marked. Also revealed is the generation of a keyhole pore. 